\title{
Dynamic and permanent gratings in suspensions of absorbing nanocrystals in an organic solvent
}

\author{
S. Kredentser, ${ }^{1}$ A. Eremin, ${ }^{2}$ P. Davidson, ${ }^{3}$ V.Reshetnyak, ${ }^{4}$ R.Stannarius, ${ }^{2}$ and Yu. Reznikov ${ }^{* 1}$ \\ ${ }^{1}$ Institute of Physics of National Academy of Sciences of Ukraine, Pr. Nauky 46, Kyiv, 03028 Ukraine \\ ${ }^{2}$ Otto-von-Guericke-Universität Magdeburg, FNW/IEP/ANP, Postfach 4120, Magdeburg, Germany \\ ${ }^{3}$ Laboratoire de Physique des Solides, UMR CNRS 8502, Université Paris-Sud, Orsay 91405, France \\ ${ }^{4}$ Taras Shevchenko National University of Kyiv, Kyiv 01601, Ukraine
}

Received November 30, 2015; accepted December 29, 2015; published December 31, 2015

\begin{abstract}
Both dynamic and permanent optical gratings were recorded in the suspension of absorbing pigment nanocrystallites in organic solvent. The dynamic gratings were recorded due to the transfer of nanocrystallites in a temperature gradient induced by a light interference pattern, and the permanent gratings were formed due to light-induced adsorption of nanocrystalites on the surface of a cell substrate.
\end{abstract}

It is traditionally considered that giant effects of lightinduced refractive index changes are characteristic of liquid crystals, and that these effects are rather weak in isotropic liquids [1]. This statement is correct in the case of traditional mechanisms of optical nonlinearity (thermal nonlinearity, orientational nonlinearity, and photo-transformation nonlinearity), but isotropic liquid suspensions also reveal a strong optical nonlinearity due to the thermophoresis of absorbing particles in a solvent. The movement of the particles in a temperature gradient due to the absorption of spatially modulated light irradiation (light-induced thermophoresis or light-induced Soret effect) results in the redistribution of particle concentration in the liquid matrix. Since the refractive index of the suspension, $n_{\text {susp }}$, and the absorption coefficient, $\beta_{\text {susp }}$, are proportional to the concentration of particles, $c_{\text {part }}$, the spatial modulation of particle concentration results in a modulation of $n_{\text {susp }}$ and $\beta_{\text {susp }}$. This opens up an application opportunity for the lightinduced Soret effect in developing highly-sensitive nonlinear optical media.

The light-induced Soret effect was first observed by Thyagarajan and Lallemand in 1978 [2]. Bloisi et al. suggested that this effect could be used for recording concentration amplitude and phase optical gratings [3]. Later, the recording of such dynamic gratings was reported by Köhler [4]. The light-induced Soret effect in different kinds of suspensions was also studied by the non-linear lens method [5-7]. Dynamic optical gratings and nonlinear lenses were used for the measurements of Soret coefficients and thermo-optical coefficients.

*E-mail: yurireznikov@hotmail.com
Here, we present the study of the recording effective phase and amplitude gratings in a suspension of absorbing elongated nanocrystallites. We observed not only dynamic but permanent gratings in this suspension. The permanent gratings were found to be due to light-induced adsorption of nanocrystallites on the substrates of the cell filled with the suspension.

The gratings were recorded in a suspension of elongated nanocrystals of Pigment Red 176 (Novoperm Carmine HF3C, Clariant, Germany) in dodecane. The production of these nanoparticles and their suspension in dodecane are described in detail in [8]. The length of the nanoparticles was $230 \mathrm{~nm} \pm 70 \mathrm{~nm}$ and their diameter was $46 \mathrm{~nm} \pm 20 \mathrm{~nm}$. The nanocrystals are photo-stable and absorb light in a green part of the visible spectrum (the absorption coefficient of the suspension is $\beta=3 \cdot 10^{5} \mathrm{~m}^{-1}$ at an optical wavelength $\lambda=532 \mathrm{~nm}$ ). The volume fraction of the nanoparticles was $c_{v}=2.4 \%$. The suspension was isotropic at this concentration (the transition to a nematic phase occurs at $c_{v} \approx 15 \%$ ) [8].

The suspension was confined in cells made from two glass substrates, the inner surfaces of which were covered with transparent ITO electrodes. The cells of the thickness $L=20 \mu \mathrm{m}$ were sealed with epoxy glue.

We used a standard setup for recording dynamic holograms. Two linearly polarized YAG laser Gaussian beams of equal intensities $(\lambda=532 \mathrm{~nm})$ and parallel polarizations overlapped in the plane of the cell, creating a sinusoidal interference pattern with a spatial intensity variation $I=I_{0} \cdot(1+\cos q x)$, where $q=2 \pi / \Lambda$ is the grating vector and $\Lambda=10 \div 50 \mu \mathrm{m}$ is the period of the interference pattern. The diameter of the beams in the plane of the interference pattern was $d=0.34 \mu \mathrm{m}$.

The holograms were monitored by photodiodes, either in the self-diffraction regime, by observation of the firstorder non-Bragg diffraction beams behind the cell, or by observation of the first-order diffraction of the probe beam of a He-Ne laser $\left(\lambda_{\text {probe }}=632 \mathrm{~nm}\right)$. The polarization of the probe beam was parallel to that of the recording beams. 
Due to the Soret effect, switching on the recording beams gives rise to the redistribution of the nanoparticles in the interference pattern and therefore - to the appearance of the self-diffraction orders and the diffraction orders of the probe. Both amplitude and phase dynamic gratings were observed in the self-diffraction regime because the beams of the recording lasers were absorbed by the suspension. The wavelength of the He-Ne laser lies in the transparency region of the suspension. Therefore, only the phase grating was monitored by the probe laser. In this case, the diffraction efficiency of the grating reads:

$$
\eta_{\text {probe }}=\frac{I_{d}^{ \pm 1}}{I} \approx\left(\frac{\pi \Delta n_{\text {susp }} L}{\lambda_{\text {probe }}}\right)^{2},
$$

where $\boldsymbol{I}_{d}^{ \pm 1}$ is the intensity of the first order diffraction beams and $\Delta n_{\text {susp }}$ is the light-induced change of an refractive index of the suspension at the maximum of the interference pattern.

For typical values $I \approx 65 \mathrm{~W} \cdot \mathrm{cm}^{-2}$ and $\Lambda=40 \mu \mathrm{m}$, the diffraction efficiency was $\eta_{\text {probe }} \approx 2 \%$. In first approximation, the modulation $\Delta n_{\text {susp }}$ is proportional to the amplitude of the modulation of particle concentration, $\Delta c_{v} \sim I$ and $\Delta n_{\text {susp }}=n_{2} I$, where $n_{2}$ is the non-linear parameter of the suspension. For $I \approx 65 \mathrm{~W} \cdot \mathrm{cm}^{2}$ and $\Lambda=40 \mu \mathrm{m}$, $\Delta n_{\text {susp }} \approx 10^{-3}$ and $n_{2} \approx 10^{-2} \mathrm{~kW} \cdot \mathrm{cm}^{-2}$. These values are very high; the value $n_{2}$ is actually of the same order as that of molecular thermotropic liquid crystals whose nonlinearities are called "giant' [1].

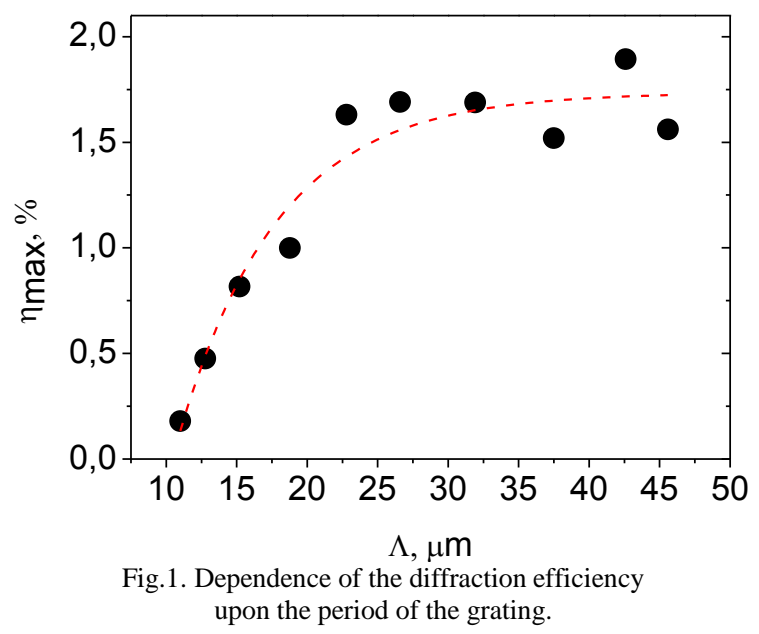

The diffraction efficiency, $\eta_{\text {probe }}$, depends on the period of the grating. It increases almost linearly with the period of the grating up to the values comparable with the cell thickness $L=20 \mu \mathrm{m}$, and then saturates (Fig.1).

The dynamics of grating recording and relaxation after switching on and off the recording beams is presented in Fig. 2. If the recording beams are switched off right after reaching the steady-state value of $I_{d}$, the dynamics is well described by single exponential laws with the characteristic times $t_{o n}=29 \mathrm{~s}$ and $t_{\text {off }}=15 \mathrm{~s}\left(I \approx 65 \mathrm{~W} \cdot \mathrm{cm}^{2}\right.$ and $\Lambda=40 \mu \mathrm{m})$ although the dynamics were also well fitted with two exponential functions in some experiments. Both the recording and relaxation characteristic times almost linearly increased with the period of the grating, in the accessible range $\Lambda=10 \div 45 \mu \mathrm{m}$.

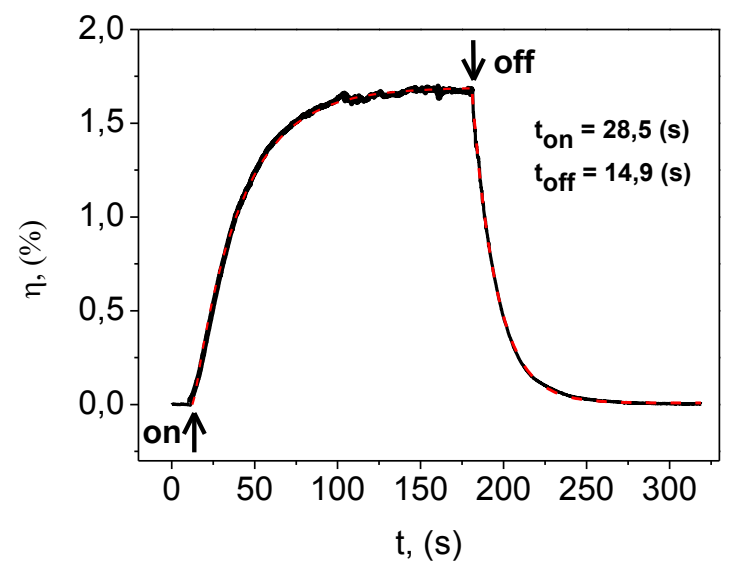

Fig.2. The diffraction efficiency of the grating upon switching the recording beams on and off.

It should be noted that the inequality $t_{o n}>t_{\text {off }}$ is very uncommon. Usually, the recording time is either equal or shorter than the relaxation time because of an accelerating drag force during the recording [9]. We suggest here that the unusual dynamics could be related to the anisotropic rod-like shape of the nanocrystals. Indeed, the characteristic times of the grating recording and relaxation are proportional to the time required for nanoparticles to diffuse parallel to the grating vector over a distance of the order of $\Lambda / 2$. This time is inversely proportional to the diffusion coefficient of the nanorods in dodecane. The diffusion of hard rods in a dense (nematic) colloid depends on their orientational order parameter, $S$, $D_{\|} / D_{\perp} \approx(2 S+1) /(1-S)$, where $D_{\|}$and $D_{\perp}$ are the diffusion constants parallel and perpendicular to the anisotropy axis [10]. At the beginning of the recording, the colloid is isotropic, $S=0$, and the diffusion is determined by the average diffusion constant, $\dot{D}=D_{\|} / 3+$ $+2 D_{\perp} / 3$. During the recording, the nanorods are aligned in the flow due to the Soret effect. Therefore, the subsequent relaxation is governed by the constant $D_{\|}>\dot{D}$ and the relaxation is therefore faster than the recording. Since

$$
\frac{t_{\text {on }}}{t_{\text {off }}}=\frac{D}{2 D_{\perp} / 3+D_{\downarrow} / 3} \approx 2,
$$

the ratio

$\frac{D_{\square}}{D_{\perp}}=\frac{2 S-1}{1-S} \approx 4$ 
and the estimation flow-induced order parameter has a reasonable value $S \approx 0.5$.

If the recording beams remain switched on for a long time (minutes), the intensity of the diffracted beams decreases and the grating eventually disappears. Evidently, this is due to a complete light-induced transfer of nanoparticles to the periphery of the Gaussian envelope of the interference pattern.

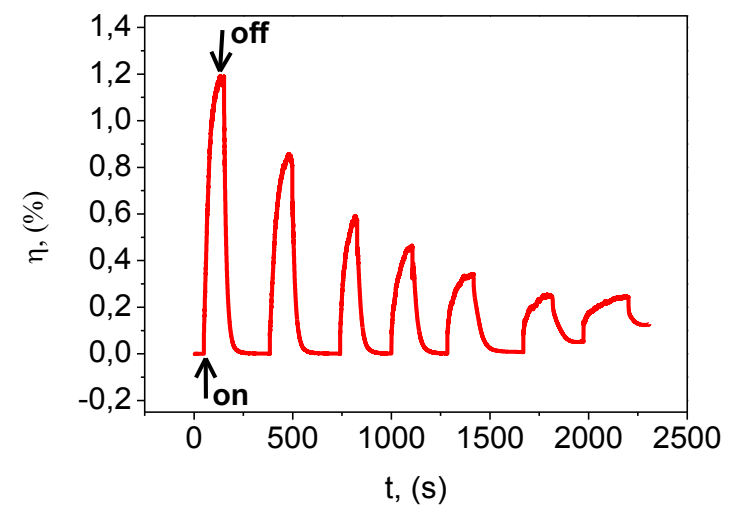

Fig.3. Cycling recording/relaxation of the gratings.

In addition to the dynamic gratings arising from the modulation of particle concentration in the bulk of the suspension, permanent gratings were also observed. To record this permanent grating, we repeatedly recorded and erased the dynamic gratings several times (Fig.3). The disassembling of the cell and removal of the suspension showed the presence of a permanent grating on the substrate (Fig. 4). The diffraction efficiency of the permanent grating is about 5-10 times lower than that of the dynamic grating.

After disassembling the cell and removing the excess suspension from the substrate by an air jet, a layer of adsorbed nanocrystals was found in the irradiated area (Fig. 4), whereas no trace of an adsorbed layer was found in non-irradiated areas. The thickness of the adsorbed layer was spatially modulated with the period of the interference pattern. Thus, permanent gratings are due to light-induced adsorption of the pigment onto the substrates. Recently, this effect was observed and studied by us on irradiation of the suspension with a Gaussian beam [11].

To conclude, the light-induced Soret effect was applied for recording dynamic holograms. The particles transfer in the temperature gradient brings about strong changes of the absorption coefficient and refractive index of the suspension. The corresponding large non-linear optical coefficient is comparable to that of nematic liquid crystals and allows recording efficient optical gratings with low-power continuously operating lasers.

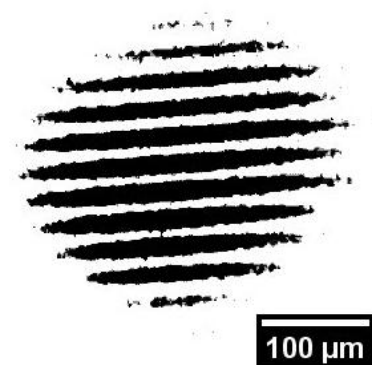

Fig.4. Permanent grating formed by the light-induced adsorbed layer of nanocrystallites on the cell substrate.

The authors acknowledge financial support from DAAD within the Ukrainian-German scientifictechnological collaboration grant "Magneto-optical anisotropic suspensions of dye nanocrystallites", and by DFG within the Project STA425/36-1 in SPP 1681.

\section{References}

[1] B.Y. Zel'dovich, N. V Tabiryan, Sov. Phys. Uspekhi 28, 1059 (1985).

[2] K. Thyagarajan, P. Lallemand, Opt. Commun. 26, 54 (1978).

[3] F. Bloisi, L. Vicari, P. Cavaliere, S. Martellucci, J. Quartieri, P. Mormile, G. Pierattini, A. Felice, Appl. Phys. B 106, 103 (1987).

[4] W. Köhler, Chem. Phys. 98, 660 (1993).

[5] J. Zamir Anvari, R. Karimzadeh, N. Mansour, J. Opt. 12, 035212 (2010).

[6] N.M. Rouhollah Karimzadeh, J. Mod. Opt. 60, 163 (2013).

[7] N. Tabiryan, W. Luo, Phys. Rev. E 57, 4431 (1998).

[8] A. Eremin, R. Stannarius, S. Klein, J. Heuer, R.M. Richardson, Adv. Funct. Mater. 21, 556 (2011).

[9] I.C. Khoo, Phys. Rep. 471, 221 (2009).

[10] H. Löwen, Phys. Rev. E 59, 1989 (1999).

[11] S. Kredentser, A. Eremin, P. Davidson, V. Reshetnyak, R. Stannarius,

[12] Y. Reznikov, submitted to Eur. Phys. J. E (2015). 